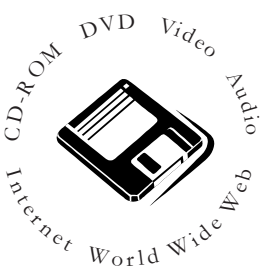

\section{Basics of Web-Page Design for Use in Medical Education}

\author{
(Medical Education Web Page Series - Part 4) \\ [1] What is different about Medical Education Web \\ pages?
}

In most respects, Medical Education Web pages are no different than any other kind of well-designed Web page. However, there is an expectation that Medical Education Web pages should have a high level of professionalism, with an emphasis on providing high quality content, with good formatting and good use of visuals, and, above all, with a special effort made to ensure that the contents are highly accurate, especially when providing critical information such as drug doses. ${ }^{1}$ In addition, Medical Education Web pages should ideally follow the "Health on The Net" guidelines, ${ }^{2}$ discussed next.

\section{[2] HON guidelines}

The Health On the Net Foundation (HON, http://www.hon.ch), created in 1995, is a not-forprofit International Swiss Organization with a mission to guide users to quality online medical information and to provide ethical standards for Web site developers. MedHunt, HONselect and the HON Code of Conduct (HONcode) are three services offered by HON. The first initiative, MedHunt, "consists of an intelligent and specialized search engine designed to locate Internet information related to a given medical and health domain." The second service, HONselect, "is the first assisted-search facility that integrates heterogeneous databases to offer users a full assortment of healthcare information and resources available on the Web." The third service "is an authoritative set of voluntary guidelines designed to raise the quality of Webbased medical and health information." Table I below outlines the HON principles.
TABLE I HON principles (from www.hon.ch)

\section{Authority}

Any medical or health advice provided and hosted on this site will only be given by medically trained and qualified professionals unless a clear statement is made that a piece of advice offered is from a non-medically qualified individual or organization.

\section{Complementarity}

The information provided on this site is designed to support, not replace, the relationship that exists between a patient/site visitor and his/her existing physician.

3. Confidentiality

Confidentiality of data relating to individual patients and visitors to a medical/health Web site, including their identity, is respected by this Web site. The Web site owners undertake to honour or exceed the legal requirements of medical/health information privacy that apply in the country and state where the Web site and mirror sites are located.

\section{Attribution}

Where appropriate, information contained on this site will be supported by clear references to source data and, where possible, have specific HTML links to that data. The date when a clinical page was last modified will be clearly displayed (e.g., at the bottom of the page).

5. Justifiability

Any claims relating to the benefits/performance of a specific treatment, commercial product or service will be supported by appropriate, balanced evidence in the manner outlined above in Principle 4.

\section{Transparency of authorship}

The designers of this Web site will seek to provide information in the clearest possible manner and provide contact addresses for visitors that seek further information or support. The Webmaster will display his/her E-mail address clearly throughout the Web site.

\section{Transparency of sponsorship}

Support for this Web site will be clearly identified, including the identities of commercial and non-commercial organizations that have contributed funding, services or material for the site.

8. Honesty in advertising o editorial policy

If advertising is a source of funding it will be clearly stated. A brief description of the advertising policy adopted by the Web site owners will be displayed on the site. Advertizing and other promotional material will be presented to viewers in a manner and context that facilitates differentiation between it and the original material created by the institution operating the site. 


\section{[3] Needs assessment}

Any Medical Education Web page should be planned based on a "needs assessment". A needs assessment is simply a means of determining the educational needs of your target audience. Although in many cases this is done with a formal survey sent out to a target audience, ${ }^{3}$ ideas for topics may also be explored through "hallway discussions" with colleagues and residents and by exploring the medical literature via PubMed (http://www.ncbi.nlm.nih.gov/PubMed/) and elsewhere. In many cases the needs assessment is implicit, such as when a professor constructs a Medical Education Web page for the benefit of his or her students.

\section{[4] Educational objectives}

Regardless of the kind of page you are trying to produce, it is important that you establish from the onset what your educational objectives are. Usually, only three or four objectives need be established for each Web page you wish to produce, and these objectives should be borne in mind as the page you are producing is developed. Keeping your educational objectives in mind not only helps keep your page focused, but it also helps in getting your materials accredited, since most CME accreditation programs require that explicit educational objectives be established. As an example, here are some sample objectives that might be found on a Web page concerned with anesthesia for otolaryngology patients.

- Understand the particular surgical and anesthetic needs of the otolaryngology patient, particularly with respect to airway issues.

- Explain common anesthetic or surgical complications associated with various otolaryngology procedures.

Understand the relevant anatomy and physiology underlying successful management of otolaryngology procedures.

\section{[5] Web page elements}

A good Medical Education Web page will make use of a number of Web page elements. Although a satisfactory page can be produced using only text, the addition of forms, frames, tables, hyperlinks, graphic images, sound files and video clips provides the opportunity to liven up a page, making it more attractive to your target audience. These elements can also be used to add interactivity to the Website (see below). For more information on Web page elements take a look at http://www.htmlgoodies.com.

\section{[6] Page layout, structure and esthetics}

The organization of a Website will influence its success or failure. The "look" of a site is one of the key factors for how a user perceives the effort behind it. Web pages must be easy to read, and easy to navigate, that is - the reader must understand where to click next. There are many design conventions that should be followed to make a visitor's experience valuable. The design of the site should also support the educational goals. Finally, remember that Web pages are living documents that require at least occasional updating.

One simple way to check the technical quality of your Web site is to use a service called Tune Up, a service of Web Site Garage (http://website garage.netscape.com). It offers the following checks for your site:

- Browser Compatibility - to verify that your Web site will display correctly in different browsers and versions.

- Registration Readiness - to make sure your site is ready to be indexed and submitted to the various search engines and directories.

- Load Time Check - reports load time for various connection speeds.

- Dead Link Check - to detect dead links.

- Link Popularity Check - to find out how many sites are linking to you.

- Spell Check - to catch misspelled words.

Finally, note that one particularly convenient and easy way to construct simple but esthetically pleasing Web pages is to start with a single-cell table, say 600 pixels wide. Then simply put all of the Web page elements into the table. This is most easily done using a program like Microsoft FrontPage.

\section{[7] Use of visuals}

Visual communication, most educators agree, helps add interest and even excitement to a topic. Some experts believe that humans are genetically wired to communicate visually and that images are often more effective than words in cognitive processes - basically a neurophysiological version of the proverb that "a picture is worth a thousand words." Indeed, Professor Semir Zeki of the Institute of Cognitive Neuroscience at the University College, London (http:// www.icn.ucl.ac.uk/members/Zeki89/) has theorized about the "visual brain" and development of spatial awareness, abstract thought, and what he calls "neuroesthetics" - the study of the neural basis for perception, creativity, and achievement. All this serves to restate what almost everyone who teaches already knows - that most people are visual learners, at least to some degree.

Still, it is important to bear in mind that highly visual Web pages can sometimes take a long time to download over ordinary telephone dialup connec- 
TABLE II Web page evaluation criteria

Source: http://www.uxl.eiu.edu/ cfmgb/web.htm. See also other Web page evaluation worksheets and criteria available at http://www.duke.edu/ del/evaluate.html, at http://www.vuw.ac.nz/ agsmith/evaln/ and at http://lib.nmsu.edu/instruction/evalcrit.html

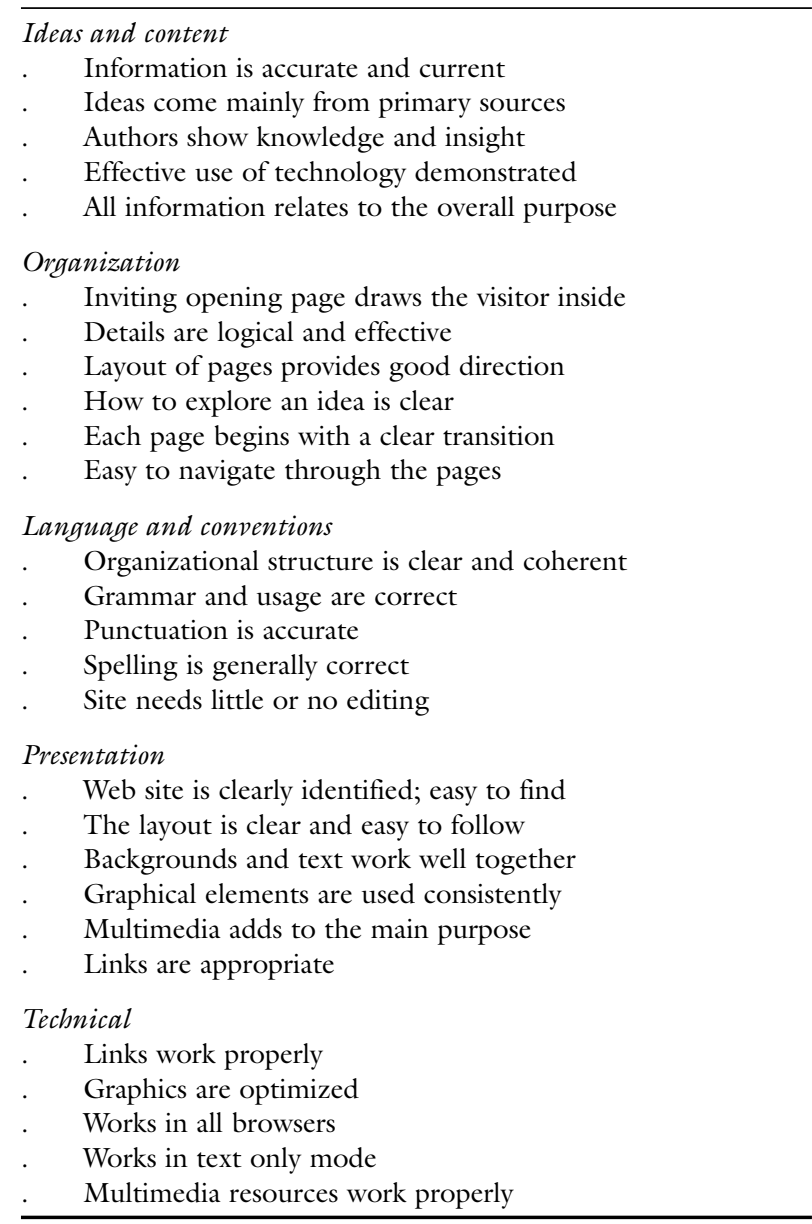

tions. One way to help determine if your images are optimized for low-speed communications is to use GIF Lube, another service of Web Site Garage. Use of GIF Lube will help decrease your image load times by optimizing your graphics. It is simple to use: just type in the URL of your site, or the image, and it will immediately compress the image and give you back the results.

\section{[8] Interactivity}

Adult learning theory emphasizes the importance of interactivity in the educational process. In exploring the differences between traditional teaching methods and education via the Web, the issue of differences in the patterns and properties of interaction in the two settings figures prominently. Students using the Web for distance education may interact variously with the instructor, the content, the technology, and with other students, but interaction with technology is especially important in Web-based distance education. In this respect, good design principles must be adhered to for successful outcomes. This view is supported by a landmark meta-analysis of the effectiveness of formal CME by Davis et al. ${ }^{4}$ who showed that traditional didactic methods do not generally lead to a change of clinical practice, or to an improvement in patients' health outcomes, whereas interactive techniques are more likely to. This important issue will be dealt with in more detail in a future article.

\section{[9] Web page evaluation}

The use of Web page evaluation criteria should be born in mind at all stages of Web page development. Table II provides a useful set of criteria that can be used to evaluate the various drafts of your effort. In addition, specific student evaluations can be helpful in ensuring that students find your educational Web site to be valuable. Although a structured questionnaire is commonly employed for this purpose, informal discussions with students can also be quite helpful.

\section{[10] Publicize your site}

Once your Web site has been launched, you will want to use promotion tools to register your site with the hundreds of search engines and directories that exist. Search engines such as Google (http://google.com), AltaVista (http://www.altavista.com), Excite (http://www.excite.com), and Hotbot (http:// www.hotbot.com) index the contents of Web pages for the benefit of people who want to search the Internet. They do so by sending out web crawlers software robot programs that automatically find and retrieve Web pages and add the information to their indexes. Thus, if your Web site is new, there are probably very few links to it, and it could take months for the web crawlers to find you on their own.

You can help speed up the process. Near the bottom of each search engine page, there is usually an "Add URL" icon. Click on that, and then enter the URL for your site. This way your information will be indexed much faster.

However, there is an even faster way. One can use one or more of the services listed below to have your site submitted to a number of search engines. However, many of these services are not free.

- http://www.addme.com/

- http://www.submit-it.com/

- http://www.webcom.com/html/publicize.shtml

- http://www.netpost.com 
- http://www.register-it.com/

- http://www.webpromote.com/

One particularly valuable means to publicize medical education sites is to join the Medical Education Web Ring, which is a series of sites dedicated to medical education. It includes both educational resources and sites that index medical education Web pages. Check it out at http://www.med.jhu.edu/medcenter/mer/. If you have a medical education-related page, joining the ring will bring you more visitors. Similarly, people who frequent your site can use it to find more pages of similar nature.

\section{References}

1 Kiley R. Medical education on the World Wide Web. J R Soc Med 1998; 91: 307-9.

2 Boyer C, Selby M, Appel RD. The health on the net code of conduct for medical and health web sites. Medinfo 1998; 9(Pt 2 ) :1163-6.

3 Broomfield D, Humphris GM. Using the Delphi technique to identify the cancer education requirements of general practitioners. Med Educ 2001; 35: 928-37

4 Davis D, O'Brien MA, Freemantle N, Wolf FM Mazmanian P, Taylor-Vaisey A. Impact of formal continuing medical education: do conferences, workshops, rounds, and other traditional continuing education activities change physician behaviour or health outcomes? JAMA 1999; 282: 867-74.

\section{Erratum}

In the article entitled: "Platelet count may predict abnormal bleeding time among pregnant women in hypertension and preeclampsia" - Can J Anesth 2001; 48: 563-9, the Results section of the abstract, should have read: "A platelet count less than or equal to $75 \times$ $109 / \mathrm{L}$ was specific for the presence of an abnormal BT..."

Please accept our apologies for any inconvenience. 